\title{
OTIMIZAÇÃO DOS MEIOS FILTRANTES DE FILTRO DE MANGAS EMPREGADOS NA INDÚSTRIA QUÍMICA
}

\author{
F.M. OLIVEIRA ${ }^{1}$, J.C. REIS ${ }^{1}$ e M.L. AGUIAR ${ }^{1}$ \\ ${ }^{1}$ Universidade Federal de São Carlos, Departamento de Engenharia Química \\ E-mail para contato: flavinhamatias@hotmail.com
}

\begin{abstract}
RESUMO - A preocupação com o meio ambiente vem intensificando as pesquisas em filtração de gases, na separação gás-sólido. A finalidade destas pesquisas é obter métodos mais eficientes e com menor gasto energético. Neste sentido, o presente estudo objetivou analisar a influência do carregamento corona na formação da torta de filtração de gases em filtro de mangas, para melhorar a eficiência da coleta das partículas. Para isso adaptou-se um sistema de filtração de gases, existente no DEQ-UFSCar, para receber um carregador corona, posteriormente obteve-se o ponto ótimo de operação a uma vazão mássica de 9 $\mathrm{mg} / \mathrm{s}$ e velocidade de filtração de $10 \mathrm{~cm} / \mathrm{s}$, variando-se a tensão da unidade entre $0,-1,0$ e $-2,0 \mathrm{kV}$. Através dos ensaios realizados foi possível confirmar que, com a aplicação da corrente corona, a eficiência de coleta das partículas aumentou para uma menor perda de carga.
\end{abstract}

\section{INTRODUÇÃO}

$\mathrm{Na}$ tentativa de se obter equipamentos mais eficientes na área de filtração de gases, novas fibras vêm sendo desenvolvidas ou aprimoradas, para coletar partículas cada vez menores, chegando a se obter uma alta efíciência até mesmo para as nano partículas.

Segundo Midha (2011), entre os diversos tipos de meios filtrantes os filtros de tecidos são os mais eficientes. Os filtros trançados e não trançados são os mais utilizados na filtração de gases. Devido as características das fibras dos tecidos não trançados, eles tem uma rápida formação da torta de filtração de gases.

A filtração de gases por meio do uso de filtros de tecido, frequentemente formado por fibras sintéticas, permite a passagem do fluido com alta retenção do material particulado. À medida que a filtração ocorre as partículas se acumulam no filtro e formam a chamada torta de filtração. O acúmulo desta torta aumenta a eficiência do processo de filtração, uma vez que ela provoca a diminuição da porosidade do filtro. No entanto, isso também dificulta a passagem do ar gerando um aumento do gasto de energia para manter o fluxo gasoso em níveis operacionais.

Vários trabalhos estão sendo realizados no Departamento de Engenharia química da UFSCar 
visando avaliar o processo de formação da torta de filtração de gases e a sua interação com o filtro.

Estudos como os de Fenara et. al (2011) e Rodrigues et. al (2011) mostraram que com a aplicação da carga eletrostática nas partículas, a eficiência de coleta do material particulado aumentou e a perda de carga do sistema diminui. Esta redução da perda de carga leva a uma economia de energia, além de prolongar o tempo de filtração de gases, bem como melhorar o processo de limpeza das mangas, ocasionando benefícios para as indústrias.

As cargas das partículas podem ocorrer espontaneamente por atrito ou choque (triboeletrificação ou impacto) entre elas, inerentes ao processo de filtração, ou podem ser deliberadamente introduzidas, por exemplo, por corrente corona (Meira, 2009).

O carregamento por corrente corona é decorrente de duas etapas. A primeira, em que ocorre o processo de ionização do gás, e a segunda, dada pelo carregamento das partículas.

$\mathrm{Na}$ ionização, ocorre uma grande produção de íons em fase gasosa por meio de um campo elétrico de alta intensidade obtido através do uso de altos potenciais aplicados a um eletrodo de descarga (Rodrigues, 2006). Dessa forma, a ionização decorre da retirada de elétrons das moléculas neutras de gás por meio de impacto com elétrons livres movidos pelo campo elétrico. $\mathrm{Na}$ corona negativa os íons positivos se movem em direção ao eletrodo de descarga.

É importante ressaltar que a concentração e o diâmetro das partículas podem influenciar no efeito de carregamento corona. Meira (2009), estudando nanofiltração por precipitação eletrostática, verificou eficiência acima de $99 \%$, para velocidades de 1 a $10 \mathrm{~cm} / \mathrm{s}$, e constatou a tendência de aumento da eficiência com o decréscimo do diâmetro da partícula filtrada.

Com os resultados favoráveis obtidos nos trabalhos anteriores, verificou-se a possibilidade de otimizar o processo de filtração, o que incentivou dar continuidade a esta linha de pesquisa variando o tamanho da partícula e a voltagem do efeito corona. Portanto, esse trabalho teve por objetivo investigar o comportamento das partículas, durante a formação da torta de filtração de gases, após serem carregadas eletrostaticamente.

\section{MATERIAL E MÉTODOS}

\subsection{Equipamentos}

Para realização dos ensaios experimentais de filtração com carga eletrostática e sem carga, foi utilizado o equipamento localizado no Laboratório de Controle Ambiental do Departamento de Engenharia Química, da Universidade Federal de São Carlos (UFSCar), ilustrado na Figura1.

A unidade consiste de um sistema de geração e controle de umidade do ar, sistema de dispersão do pó, suporte de filtração, equipamentos de medida e sistema de aquisição de dados. 


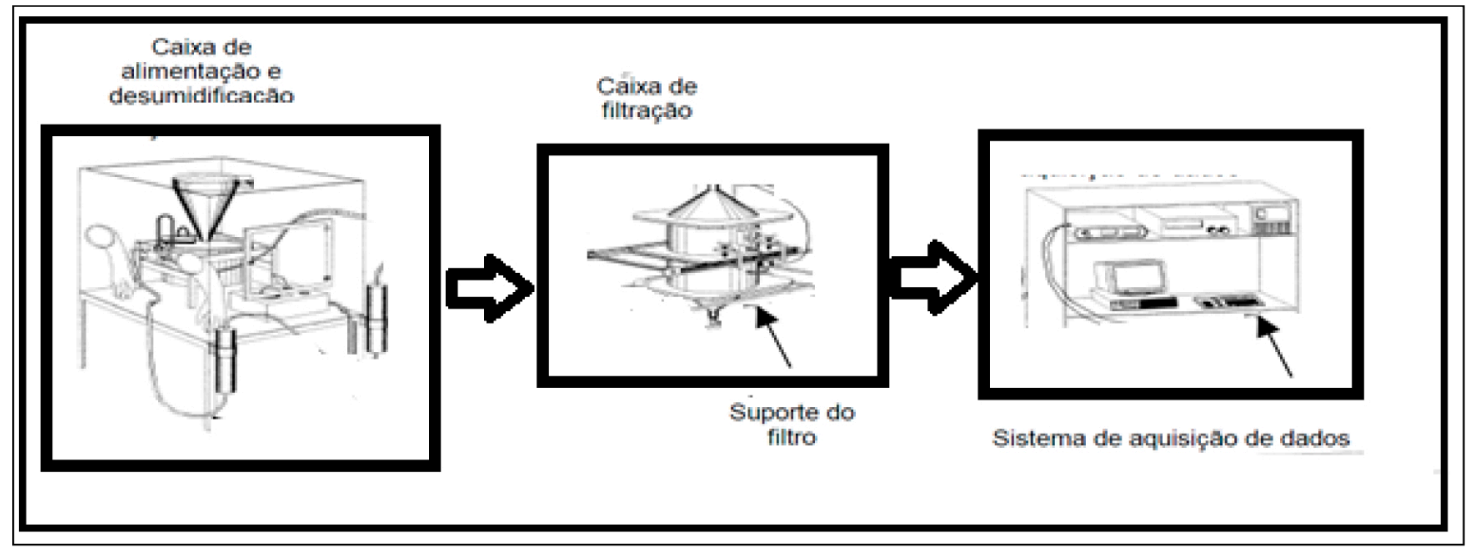

Figura 1 - Unidade experimental sem o carregador corona.

O aparato experimental foi construído para simular a operação de filtração no laboratório. Essa unidade foi adaptada, para receber o carregador corona localizado entre a caixa de alimentação e a caixa de filtração, demostrado na Figura 2.

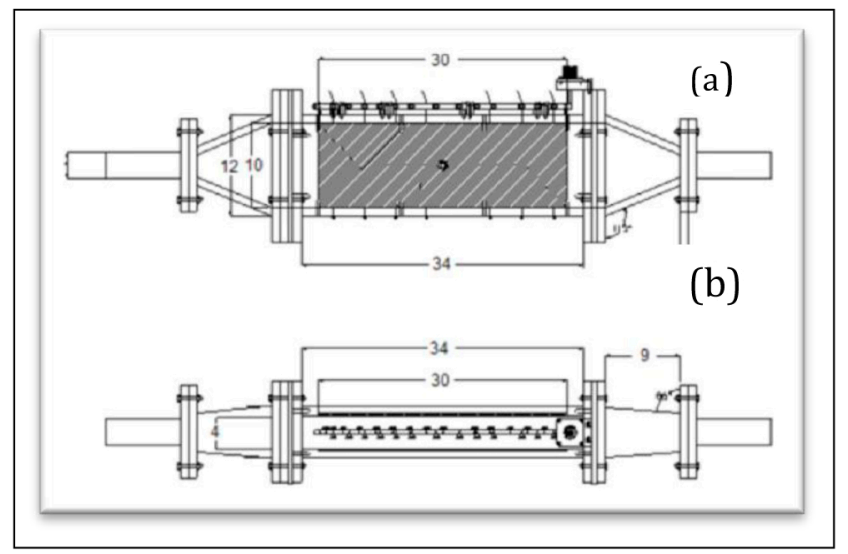

Figura 2 - Sistema de carregamento corona com unidade das medidas em $\mathrm{cm}$. (a) Vista frontal, onde a região hachurada representa a placa de cobre (eletrodo de coleta). (b) Vista superior, no centro encontra-se o suporte metálico dos fios de cobre que possui a função de distribuir corrente elétrica entre os mesmos.

O carregador corona consiste de um precipitador eletrostático tipo placa-fio que foi utilizado com o intuito de apenas carregar as partículas eletrostaticamente, via efeito corona e não para arrastá-las como ocorre na filtração por precipitação. A unidade de carregamento das partículas é composta por uma caixa retangular de acrílico com cinco fios de cobre alinhados no centro, os quais funcionam como eletrodos de descarga de um carregamento corona. O eletrodo de coleta é composto por duas placas de cobre nas laterais da caixa de acrílico, as quais são aterradas através de ligação com fios de cobre revestidos. 


\section{$\underline{\text { Material particulado }}$}

Utilizou-se como material particulado o cimento, este foi fornecido pela Votorantim Cimentos S.A. de Itaú de Minas (MG) e é comercializado para área de construção civil em todo pais. Este material foi escolhido devido a sua grande aplicação no mercado.

Determinou-se a densidade pelo sistema Accupyc 1330 V3.03 e o valor obtido foi de $3,1 \mathrm{~g} / \mathrm{cm}^{3}$. Analisou-se o pó, cimento, com duas granulometrias diferentes, como apresenta a Figura 3, sendo uma com diâmetro médio volumétrico de $21,5 \mu \mathrm{m}$ e a outra com diâmetro médio de $8 \mu \mathrm{m} .$.

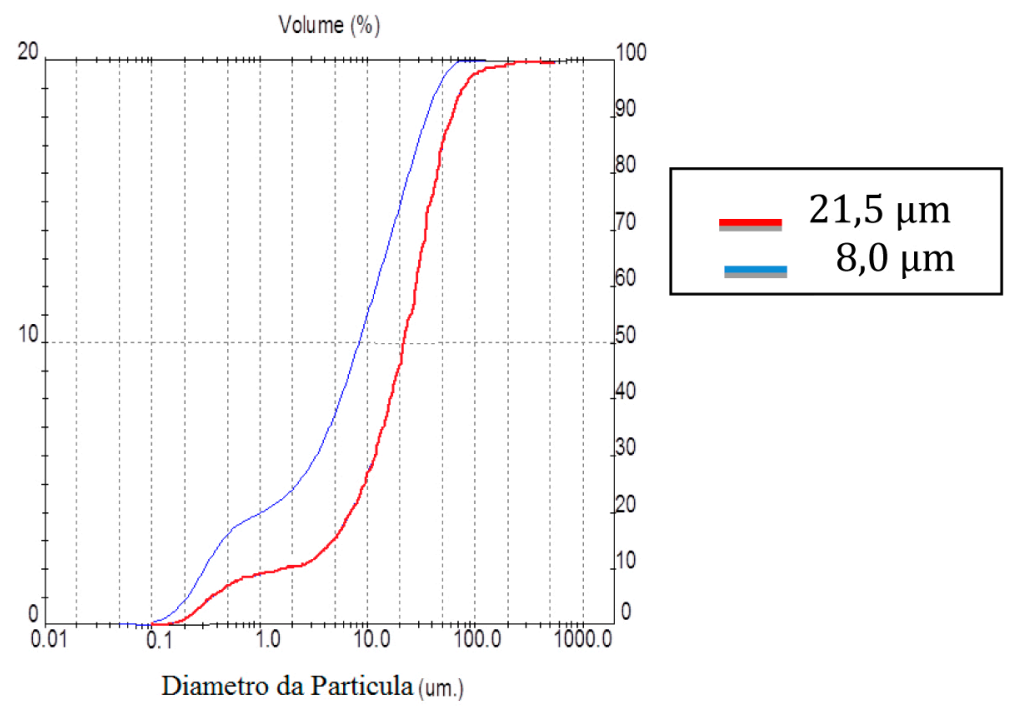

Figura 3 - Distribuição granulométrica do cimento antes do início dos ensaios.

\section{$\underline{\text { Meios Filtrantes }}$}

O meio filtrante, utilizado durante os ensaios de filtração, foi o filtro de mangas fornecido pela Votorantim Cimentos S.A, de Itaú de Minas, produzido pela BWF, Itália, de fibra de vidro, com 15,2 cm de diametro e $7 \mathrm{~m}$ de comprimento. Este foi recortado em moldes circulares de aproximadamente $0,2 \mathrm{~m}$ de diâmetro e área de filtração de $0,0254 \mathrm{~m}^{2}$, pesando aproximadamente $20 \mathrm{~g}$. Esse procedimento foi necessário para adaptá-lo no equipamento para receber a torta de filtração no laboratório.

\section{Metodologia}

As curvas de filtração foram obtidas por aquisição de dados de queda de pressão no filtro em 
função do tempo até que a perda de carga máxima do sistema atingisse, aproximadamente, 100 $\mathrm{mmH}_{2} \mathrm{O}$.

Os experimentos foram realizados a partir de testes variando as tensões de 0 a $-2 \mathrm{kV}$, para a velocidade de filtração de $10 \mathrm{~cm} / \mathrm{s}$ e vazão mássica de operação a $9 \mathrm{mg} / \mathrm{s}$. O material particulado era depositado em um sulco do prato giratório, que alimentava o filtro, que era homogeneizado durante toda a operação filtração. Inicialmente, o fluxo de gás passava em dois tubos de PVC contendo ś́lica gel, para retirar o excesso de umidade. O ar, com aproximadamente, $20 \%$ de umidade, era forçado a passar pelo prato giratório contendo o material particulado, ou seja, o cimento. Posteriormente, as partículas transportadas pelo fluxo de ar seco eram carregadas eletrostaticamente através da caixa acoplada ao sistema. As partículas carregadas depositavam-se no filtro, formando a torta de filtração de gases. Dessa forma, registrava-se a queda de pressão à medida que se formava a torta. Ao final da filtração pesava-se o meio filtrante juntamente com a torta, para estabelecer os parâmetros de estudo.

Os ensaios de filtração consistiam em simplesmente realizar testes sem carga $0 \mathrm{kV}$ (fonte de alta tensão desligada) e outros com a aplicação de $-1,0$ a $-2,0 \mathrm{kV}$ de tensão. Levando-se em conta, as variáveis que constam na Tabela 1.

Tabela 1- Parâmetros de ensaio.

\begin{tabular}{|l|c|c|c|c|c|c|}
\hline $\begin{array}{l}\text { Diâmetro médio } \\
(\mu \mathrm{m})\end{array}$ & \multicolumn{3}{|c|}{21,5} & \multicolumn{3}{c|}{8,0} \\
\hline Carga $(\mathrm{kV})$ & sem & -1 & -2 & sem & -1 & -2 \\
\hline T in $\left(\mathrm{C}^{\circ}\right)$ & 22,7 & 22,7 & 22,7 & 22 & 22 & 22 \\
\hline $\mathrm{H}$ in $(\%)$ & 20,0 & 20,0 & 20,0 & 20,0 & 20,0 & 20,0 \\
\hline Massa retida (g) & 1,7 & 2,8 & 9,8 & 2,6 & 3,2 & 2,2 \\
\hline
\end{tabular}

\section{RESULTADOS E DISCUSSÕES}

Com as variáveis apresentadas na Tabela 1 foi possível obter os gráficos de perda de carga em função da massa depositada, ou seja, as curvas de filtração, apresentados nas Figuras 4 e 5.

Verifica-se na Figura 4, para partículas de 21,5 $\mu \mathrm{m}$, que com a aplicação da carga de -1,0 e $2 \mathrm{kV}$, maior quantidade de massa foi coletada durante a filtração, para uma mesma perda de carga. Ou seja, enquanto que para partículas sem carga, foi coletada $1,7 \mathrm{~g}$, para partículas com carga de $1,0 \mathrm{kV}$, foram coletadas $2,8 \mathrm{~g}$, e para $-2 \mathrm{kV} 9,8 \mathrm{~g}$.

Portanto, para partícula de $21,5 \mu \mathrm{m}$ e carregadas, o filtro saturou com maior tempo de filtração. No entanto, esse mesmo comportamento não foi verificado para partículas de $8,0 \mu \mathrm{m}$, como pode ser verificado na Figura 5. Observa-se que, com a aplicação das cargas a massa 
coletada não teve grandes variações, ficando em torno de 2,6 para $0 \mathrm{kV} ; 3,2 \mathrm{~g}$ para $-1 \mathrm{kV}$, de $2,2 \mathrm{~g}$ para $-2 \mathrm{kV}$. Deste modo, com redução do diâmetro da partícula, diminui a retenção de partículas no meio filtrante, comparado com o que ocorreu na Figura 4, com um diâmetro de 21,5 $\mu \mathrm{m}$.

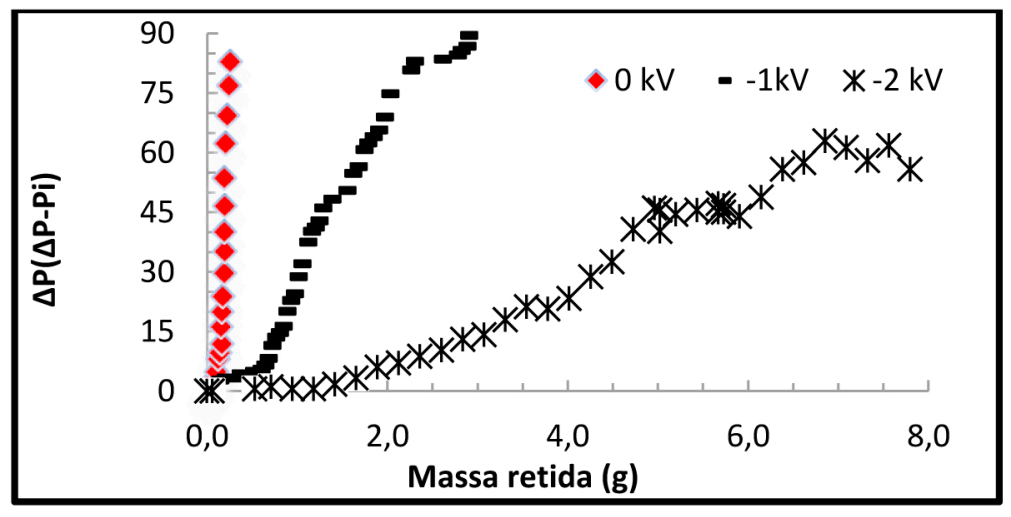

Figura 4- Curvas com partículas de 21,5 $\mu \mathrm{m}$.

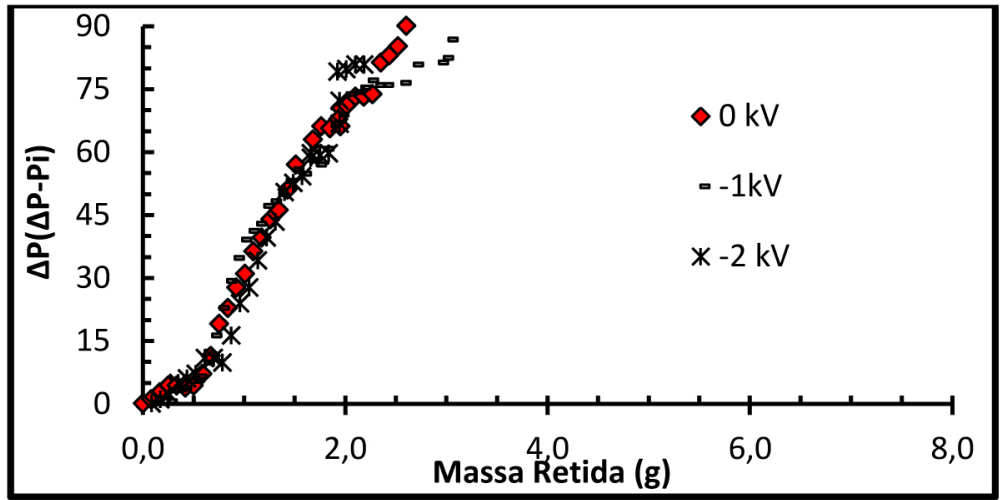

Figura 5- Curvas com partículas de $8,0 \mu \mathrm{m}$.

Comparando as Figuras 4 e 5, verifica-se que as curvas alteraram muito de um diâmetro para outro. Para o diâmetro maior, o efeito da carga foi mais evidente, aumentando o tempo de filtração, com a aplicação da carga. Já para o diâmetro menor, esse efeito foi desprezível. É bom ressaltar que foram feitas replicas dessas curvas e o comportamento se repetiu. Mas, ainda é prematuro concluir que o carregamento da partícula é influenciado pelo seu tamanho. De tal forma, a se ter um efeito positivo ou negativo na formação da torta de filtração. Ainda há necessidades de mais estudos para compreender melhor esse comportamento.

A Tabela 2 apresenta os resultados de porosidade da torta, calculado pelo Método Indireto, pela correlação de Ergun, que relaciona a queda de pressão em um leito poroso devido ao escoamento de um fluido: 


$$
\frac{\Delta P}{L}=\frac{150(1-\varepsilon) \mu U}{\varepsilon^{3} d_{p}{ }^{2}}+\frac{1,75(1-\varepsilon) \rho_{g} U^{2}}{\varepsilon^{3} d_{p}}
$$

Substituindo-se a equação $L=W / \rho_{p} \cdot(1-\varepsilon)$ e a relação $\mathrm{W}=\mathrm{Qt} / \mathrm{A}$ em $(1)$, temos a porosidade em função da perda de carga e do tempo, considerando todas outras variáveis conhecidas.

$$
\Delta P=\left[\frac{150 \cdot Q \cdot \mu \cdot U \cdot(1-\varepsilon)}{A \cdot \rho_{p} \cdot d_{p}^{2} \cdot \varepsilon^{3}}+\frac{1,75 \cdot Q \cdot \rho_{g} \cdot U}{A \cdot \rho_{p} \cdot d_{p} \cdot \varepsilon^{3}}\right] \cdot t
$$

Apresenta-se na Tabela 2 a resistência especifica da torta (LEITH e ALLEN, 1986), calculada pela Equação (3):

$$
K c=\frac{A}{V} * \frac{\Delta P}{\Delta m}
$$

Verifica-se na Tabela 2, que para os dois diâmetros estudados, com a aplicação da carga, a porosidade teórica aumentou. Ou seja, a torta ficou menos compactada, o que fez com que a perda de carga fosse menor com o tempo de filtração. Esse comportamento pode ser comprovado com os valores de resistência especifica da torta, que diminuíram com a aplicação da carga, para as partículas com diâmetro de 21,5 $\mu \mathrm{m}$. Já para as partículas com diâmetro menor, de $8 \mu \mathrm{m}$, a resistência especifica da torta reduziu para a carga de $-1 \mathrm{kV}$ e voltou a aumentar para a carga de $-2 \mathrm{kv}$.

Tabela2- Valores de porosidades e resistência específica obtidos pelo Método Indireto.

\begin{tabular}{|c|lc|lc|}
\hline \multicolumn{3}{|c|}{$\mathrm{dp} 0,5(\mu \mathrm{m}) 21,5$} & \multicolumn{2}{r|}{$\mathrm{dp0,5}(\mu \mathrm{m}) 8,0$} \\
\hline $\begin{array}{l}\text { Tensão } \\
(\mathbf{k V )}\end{array}$ & $\boldsymbol{\varepsilon}_{\text {Ergun }}$ & K2(s-') & $\boldsymbol{\varepsilon}_{\text {Ergun }}$ & K2(s-') \\
\hline 0 & 0,38 & $118 \times 10^{4}$ & 0,37 & $12,4 \times 10^{4}$ \\
-1 & 0,45 & $13,5 \times 10^{4}$ & 0,37 & $12,4 \times 10^{4}$ \\
-2 & 0,67 & $3,0 \times 10^{4}$ & 0,37 & $12,4 \times 10^{4}$ \\
\hline
\end{tabular}

\section{CONCLUSÕES}

A adaptação do sistema de carregamento corona na unidade de filtração foi realizada com sucesso. Determinando-se após a fase de testes o ponto ótimo de operação, com vazão mássica de operação a $9 \mathrm{mg} / \mathrm{s}$ e velocidade do gás a $10 \mathrm{~cm} / \mathrm{s}$. 
O diâmetro médio da partícula influenciou na perda de carga e na retenção de partículas. Porem é necessários estudos mais aprofundados na quantidade de carga aplicada nas partículas, que esta pode reter as partículas no carregador corona.

Resultados preliminares apontaram que a carga influencia na formação de torta de filtração para diferentes diâmetros. A porosidade teórica e a resistência específica da torta obtiveram resultados diferentes de uma partícula para a outra, o comportamento em partículas, para a partícula maior observou-se que aumentou a porosidade e diminuiu a resistência.

\section{NOMENCLATURA}

$A$ : área de filtração $\left[\mathrm{L}^{2}\right]$

$d_{p}$ : diâmetro médio das partículas[L]

$\varepsilon$ : porosidade do meio poroso;

$K c$ : resistência específica da torta $\left[\mathrm{t}^{-1}\right]$

$\Delta m$ : massa acumulada por unidade de área nos pontos 1 e 2 [M]
$\Delta P$ : queda de pressão nos pontos 1 e 2 da parte linear da curva de filtração [ML- $\left.{ }^{1} \mathrm{t}^{2}\right]$ $\rho_{g}:$ densidade do gás [ML-3]

$\mathrm{Q}$ : vazão mássica de dispersão do pó[ML- ${ }^{2}$ ]

$U$ : velocidade de escoamento do gás [ Lt- $\left.^{-1}\right]$

$V:$ velocidade de filtração [ $\left.\mathrm{Lt}^{-1}\right]$

$\mu$ : viscosidade do fluido $\left[\mathrm{ML}^{-1} \mathrm{t}^{1}\right]$

\section{REFERÊNCIAS}

FENARA, B. C, Rodrigues, M.V, Aguiar, M.L. Study of the electrostatic effect in the filtration of micrometer particles. São Carlos: Departamento de Engenharia Química - UFSCar, 2011. II 265 Filtech

LEITH, D.; ALLEN, R. W. K. Dust filtration by Fabric Filters. Progress in Filtration and Separation, v.4, p.1-55, 1986.

MEIRA, C. R. de. Desempenho de um precipitador eletrostático operando na remoção de nanopartículas de aerossóis. 106 p. Tese (Mestrado em Engenharia Química). UFSCar, São Carlos, 2009.

MIDHA,V.K. Filtration of gases using textile filters, Instituto Nacional de tecnologia de Jalandhar, India. Filtech, 2011.

RODRIGUES, K. B. Filtração de gases: estudo da deposição de diferentes tortas de filtração em diferentes meios filtrantes. Tese (Doutorado em Engenharia Química). Universidade Federal de São Carlos, São Carlos, 2006. 